\title{
Práticas de cuidado: o papel do enfermeiro na atenção básica
}

\author{
Care practices: the role of nurses in primary care \\ Prácticas de cuidado: el papel del enfermero en la atención primaria
}

\author{
Sonia Acioli'; Luciana Valadão Alves Kebian ${ }^{\text {II }}$; Magda Guimarães de Araujo Faria ${ }^{\text {III; }}$ \\ Patrícia Ferraccioliv ${ }^{\mathrm{IV}}$; Vanessa de Almeida Ferreira Correa ${ }^{\mathrm{V}}$
}

\begin{abstract}
RESUMO: Teve-se como objetivo conhecer as práticas de cuidado desenvolvidas por enfermeiros no âmbito da atenção básica, no município do Rio de Janeiro. Estudo de natureza qualitativa do tipo descritivo, desenvolvido entre 2009 e 2013, que teve como técnica de coleta de dados a entrevista semiestruturada com 30 enfermeiros de quatro centros municipais de saúde e duas unidades básicas de saúde da família. Os dados foram tratados utilizando-se a análise de conteúdo. Identificou-se que o enfermeiro realiza diversas práticas de cuidado na atenção básica, na qual se destacaram: consulta de enfermagem, visita domiciliar e atividades educativas. Conclui-se que o enfermeiro possui certa dificuldade em definir a presença do cuidado nas práticas que realiza, o que demonstra a necessidade de debater tais práticas e o papel do enfermeiro na atenção básica. Palavras-Chave: Enfermagem em saúde pública; cuidados de enfermagem; prática de enfermagem; prática de saúde pública.
\end{abstract}

ABSTRACT: This research aimed at assessing care practices developed by nurses in primary care in the city of Rio de Janeiro, RJ, Brazil. Qualitative study with a descriptive nature, conducted from 2009 to 2013 . Data collection was made on the basis of semi-structured interviews with 30 nurses from 04 Municipal Health Centers and 02 Family Health Basic Units. Data were analyzed on the basis of content analysis. Nurses were found to perform a broad spectrum of care practices in primary care, among which nursing consultations, home visits, and educational activities stand out. Conclusions show that nurses face difficulty defining care in their practice, which confirms the need for further discussion of practices and nurses' role in primary care. Keywords: Public health nursing; nursing care; nursing practice; public health practice.

RESUMEN: Tuvo como objetivo conocer las prácticas de cuidado desarrolladas por enfermeros de atención primaria en la ciudad de Río de Janeiro-Brasil. Estudio cualitativo y descriptivo, desarrollado entre 2009 y 2013, que tuvo como técnica de recolección de datos la entrevista semi-estructurada con 30 enfermeros de cuatro centros de salud municipales y dos unidades básicas de salud familiar. Los datos fueron tratados mediante el análisis de contenido. Se encontró que el enfermero realiza varias prácticas de cuidado en la atención primaria, con destaque para: consulta de enfermería, visita domiciliaria y actividades educativas. Se concluye que el enfermero tiene alguna dificultad para definir la presencia del cuidado en sus prácticas, lo que demuestra la necesidad de discutir tales prácticas y el papel del enfermero en la atención primaria.

Palabras Clave: Enfermería en salud pública; cuidados de enfermería; práctica de enfermería; práctica de salud pública.

\section{INTRODUÇÃO}

O enfermeiro tem na prática desenvolvida em unidades básicas de saúde (UBS) o desafio de implementar o cuidado em enfermagem na construção de relações interpessoais de diálogo, escuta, humanização e respeito. Esta prática perpassa, portanto, pela compreensão do enfermeiro sobre o significado do seu fazer profissional, ou seja, do praticar o cuidado de enfermagem na atenção básica em saúde (ABS).
Na produção científica nacional, o cuidado do enfermeiro na ABS apresenta-se como um debate a ser construído, devido à reduzida produção bibliográfica sobre o tema, o que indica a necessidade de avançar na articulação das práticas realizadas pelos enfermeiros e na reflexão sobre o cuidado. $\mathrm{Na} A B S$, a relevância de estudos sobre as práticas de cuidado realizadas por enfermeiros evidencia-se ao identificar que esta

IDoutora em Saúde Pública. Professora Adjunta do Departamento de Saúde Pública da faculdade de enfermagem da Universidade do Estado do Rio de Janeiro. Brasil. E-mail: soacioli@gmail.com.

IIEnfermeira. Mestre em Enfermagem. Doutoranda em enfermagem do Programa de Pós-Graduação em Enfermagem da Universidade do Estado do Rio de Janeiro. Brasil. E-mail: lucianavvalves@hotmail.com.

IIIEnfermeira. Mestre em Enfermagem. Doutoranda em enfermagem do Programa de Pós-Graduação em Enfermagem da Universidade do Estado do Rio de Janeiro. Brasil. E-mail: mag_araujo@yahoo.com.br.

${ }^{I V}$ Enfermeira. Mestre em Enfermagem. Doutoranda em enfermagem do Programa de Pós-Graduação em Enfermagem da Universidade do Estado do Rio de Janeiro. Brasil. E-mail: ferracciolip@gmail.com.

${ }^{\vee}$ Enfermeira. Mestre em Enfermagem. Doutoranda em Enfermagem do Programa de Pós-Graduação em Enfermagem da Faculdade de Enfermagem da Universidade do Estado do Rio de Janeiro. Brasil. E-mail: nessa aferreira@hotmail.com.

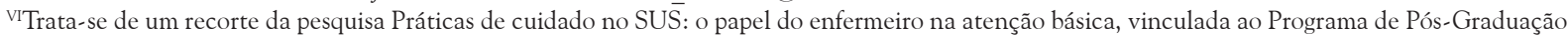
da Faculdade de Enfermagem da Universidade do Estado do Rio de Janeiro e inserido no Programa de Incentivo à Produção Científica, Técnica e Artística (Prociência). 
é considerada, pelo Ministério da Saúde, a instância prioritária e a porta de entrada para o acesso dos usuários do Sistema Único de Saúde (SUS).

Existe uma tendência das práticas de enfermagem focarem-se na doença, nos procedimentos técnicos e nas ações curativas, pouco destacando o processo de cuidar que aborda o indivíduo como centro de atenção, sendo que tal situação tem se refletido também no cuidado desenvolvido na $\mathrm{ABS}^{1}$. Dessa forma, ainda que os estudos reconheçam a atuação do enfermeiro para além da dimensão técnica, percebe-se que o núcleo de competência e responsabilidade do enfermeiro, que é o cuidado, não é usualmente abordado².

Este estudo possui como objeto a prática de cuidado do enfermeiro na ABS, entendendo-se a necessidade de pesquisas voltadas para esta temática. Além disso, parte-se do princípio de que a análise das práticas de cuidado dos enfermeiros pode fortalecer o desenvolvimento de ações interdisciplinares e integrais em consonância com as diretrizes e princípios do SUS.

Assim, teve-se como objetivo conhecer as práticas de cuidado desenvolvidas por enfermeiros no âmbito da ABS, no município do Rio de Janeiro.

\section{REVISÃo DE LITERATURA}

\section{Prática é o}

produto da relação dialética entre uma situação e um habitus, isto é, o habitus enquanto sistema de disposições duráveis é matriz de percepção, de apreciação e de ação, que se realiza em determinadas condições sociais ${ }^{3: 19}$.

Já as práticas de saúde, são construídas por meio de vários habitus e são orientadas por uma gama de influências institucionais, familiares e referentes ao grupo social de que fazem parte. Estas práticas possuem "vários sentidos, que misturam elementos da biomedicina, de outras racionalidades médicas e da chamada medicina tradicional ou popular" ${ }^{\prime: 155} \mathrm{e}$ podem ser percebidas como maneiras de fazer.

Em especial, as práticas ligadas ao cuidado são as mais próximas dos profissionais da área da saúde. O cuidado pode ser definido como velar, cuidar, tomar conta, um conjunto de ações que têm por fim e por função, manter a vida dos seres vivos com o objetivo de favorecer a reprodução e a perpetuação da vida do grupo 5 . Ele compreende mais que um momento de atenção e de zelo, pois representa uma atitude de ocupação, de responsabilidade e de envolvimento afetivo com o outro. Sendo assim, a preocupação, o respeito, a confiança, a compaixão, a solidariedade e a solicitude são algumas atitudes e comportamentos básicos do cuidado ${ }^{6}$.

O cuidado em saúde é representado pelos valores do toque, do olhar e da escuta, ultrapassando as dimensões das práticas técnicas. Nesse momento, o profissional de saúde oportuniza espaço para o diálogo, valorizando a história de vida, a crença e a cultura de cada indivíduo.

O cuidado é expressivo a partir do momento em que deixa de ser uma tarefa para ser uma ação que proporcione crescimento para quem cuida e para quem é cuidado ${ }^{7: 194}$.

Assim, para que o cuidado técnico seja considerado cuidado humano, é necessário o encontro entre o expressivo e o técnico.

O cuidado é considerado como o objeto e a essência da enfermagem, pois essa profissão envolve vário saberes, entre eles, o saber afetivo - a arte de cuidar ${ }^{6,8}$. Sabendo que o processo de cuidar relacionase com atos diferenciados, as intervenções realizadas pelo enfermeiro se caracterizam como cuidado no momento em que comportamentos de cuidar sejam exibidos, tais como o respeito, a gentileza, a atenção, a solidariedade e o interesse ${ }^{9}$.

Historicamente, o cuidado em saúde evoluiu de técnicas estritamente curativas e individualizantes para práticas integrais e coletivas. Os cuidados em saúde foram voltados basicamente para a cura das doenças, o que criava uma dicotomia entre o ser repleto de angústias, dúvidas e anseios e a patologia que ele portava. Porém, através da introdução de novos conceitos, como a Teoria Biopsicossocial, passou-se a considerar a importância dos aspectos biológicos, psicológicos e sociais do indivíduo e do coletivo e a valorizar as relações pessoais entre cliente, família e profissionais de saúde tornando, assim, a prática de cuidado em saúde mais complexa ${ }^{10}$.

O processo de cuidar envolve uma relação entre a pessoa que cuida e o sujeito, na qual o contexto socioeconômico e as singularidades políticas e culturais estão intimamente presentes ${ }^{8}$. Com isso, pode-se inferir que as práticas de cuidado do enfermeiro na atenção básica ganham significado importante, poisé neste campo que muitos contextos expressam-se claramente, refletidos através da aproximação social e cultural com a população. Consequentemente, as necessidades de saúde são identificadas in loco, o que pode proporcionar o desenvolvimento de práticas de cuidado mais coerentes e eficazes.

A partir da perspectiva de que o cuidado ultrapassa as dimensões das práticas técnicas, entende-se que espaços estimuladores do diálogo, que valorizem a história de vida, a crença e a cultura de cada indivíduo, proporcionem o desenvolvimento de práticas de cuidado. Assim, torna-se importante estudar como o enfermeiro oportuniza espaços de cuidado na ABS, uma vez que ela propõe a aproximação do profissional de saúde à realidade de vida da população.

\section{Metodologia}

Estudo de natureza qualitativa do tipo descritivo, uma vez que essa abordagem favorece a construção do conhecimento, a partir da troca de saberes entre 
entrevistador e entrevistado; o local e o global, e entre o coletivo e o indivíduo.

Os participantes da pesquisa foram 30 enfermeiros que atuam nas UBS do município do Rio de Janeiro. $\mathrm{O}$ cenário de estudo constituiu-se por quatro centros municipais de saúde (CMS) e duas unidades básicas de saúde da família (UBSF) do referido município. A pesquisa foi desenvolvida entre os anos de 2009 e 2013. A seleção dos enfermeiros se deu de modo aleatório e foi empregado o fechamento amostral por saturação de dados.

A pesquisa utilizou como técnica de coleta de dados entrevistas semiestruturadas, sendo que as entrevistas foram gravadas e posteriormente transcritas e digitadas em Word.

A técnica de tratamento de dados utilizada nesta pesquisa foi a análise de conteúdo, proposta por Bar$\operatorname{din}^{11}$. As etapas utilizadas para tratamento dos dados foram: pré-análise - onde se realizou a leitura flutuante dos depoimentos, que se constituiu no corpus da análise, seguido pela leitura exaustiva do material; análise - onde foram selecionadas as unidades de registro (frases), formando um recorte dos relatos e posterior organização. A seguir, foi construída a grade de eixos temáticos da qual emergiram as seguintes categorias: práticas de cuidado dos enfermeiros nas UBS; e dificuldades e facilidades das práticas de cuidado dos enfermeiros nas UBS. Este artigo apresenta os resultados da primeira categoria.

As entrevistas foram realizadas após a leitura e a autorização individual do Termo de Consentimento Livre Esclarecido, que garantiu o anonimato dos entrevistados. Além disso, para preservar o anonimato, os participantes enfermeiros foram identificados com a letra $\mathrm{E}$ (Enfermeiro), seguido de um número de identificação de 1 a 30. Este trabalho foi aprovado pelo Comitê de Ética em Pesquisa da Secretaria Municipal de Saúde e Defesa Civil do Rio de Janeiro, sob o protocolo n ${ }^{\circ} 32 \mathrm{~A} / 2008$.

\section{Resultados e Discussão}

A partir dos dados desta pesquisa foi possível conhecer as práticas de cuidado desenvolvidas por enfermeiros no âmbito da ABS, no município do Rio de Janeiro, e perceber que as que mais se destacam são a consulta de enfermagem, a visita domiciliar e as atividades educativas. Assim, este estudo aprofunda a reflexão sobre essas práticas de cuidado, porém destaca que nos diálogos emergiram diversas práticas, tais como acolhimento, atividades assistenciais (vacinação, realização de curativos, verificação de sinais vitais entre outros) e atividades administrativas (organização do espaço, reuniões e capacitações de equipe entre outros).

\section{Consulta de enfermagem}

A consulta de enfermagem apresentou-se como uma prática de cuidado desenvolvida pelos enfermeiros. Eles associam a consulta de enfermagem à oportunidade de realizar atividades educativas, fortalecer o vínculo, conhecer e ouvir o usuário, além de ser orientada para os conflitos, as dificuldades e a resolução dos problemas do usuário dentro de seu contexto social.

Meu preventivo [consulta de saúde da mulher] é quase uma psicologia, porque a gente discute, a gente fala. Eu não consigo colher preventivo em 20 minutos, porque ela vai falando do assunto do marido [...]. (E5)

E, aqui, dentro do nosso trabalho, a gente se aproxima dessa realidade [do usuário], a gente busca saber quais são as condições, mesmo quando ele está sendo atendido dentro do consultório. (E3)

É muito bom, nesses preventivos [consulta de saúde da mulher] eu já descobri até diabéticas. (E1)

Percebe-se a consulta de enfermagem como um espaço propício para o desenvolvimento das práticas de cuidado, pois nela o enfermeiro tem a oportunidade de ouvir demandas, avaliar as condições de saúde físicas e psicoemocionais, conhecer mais profundamente o usuário e orientar ${ }^{12}$, uma vez que ele pode utilizála para entender o contexto emocional, social e as relações familiares. Assim, este espaço favorece um cuidar que compreende mais que um momento tecnicista, mas uma atitude de envolvimento afetivo com o outro, fortalecendo o vínculo profissional-usuário ${ }^{13}$.

\section{Visita domiciliar}

Também identificou-se a visita domiciliar como prática de cuidado desenvolvida pelo enfermeiro na ABS. Tal prática está relacionada à investigação das necessidades de saúde, realização de atividades assistenciais (realização de curativos, coleta de exames, verificação de sinais vitais entre outros), escuta ativa, educação em saúde e observação da estrutura física, alimentação e relações familiares. Enfim, ela foi identificada como prática de cuidado porque proporciona espaço de atenção, diálogo, intervenção e vínculo.

Você tem que ouvir. O que está incomodando aquela pessoa, o que gerou aquilo, se aquela pessoa está com algum problema emocional na família, se é algum problema financeiro. [...] acho que o ponto fundamental ali [visita domiciliar] é ouvir, não é só falar mas também ouvir. (E2)

Através da visita domiciliar, você pode estar observando o lar dessa pessoa, não é aquela coisa de reparar, é observar. Através dessa observação, eu vejo como a pessoa está convivendo, tem gente que convive com condição de higiene precária, isso vai trazer malefícios à saúde. (E6)

O enfermeiro utiliza a visita domiciliar como estratégia de cuidado, pois além de planejar a atividade, avaliar as condições de saúde da família e propor condutas, ele desenvolve ações de modo ampliado, incluindo ao recorte individual biológico o contexto social, numa perspectiva longitudinal da atenção ${ }^{14}$. 
Mesmo diante da importância dessa prática para o planejamento de atividades voltadas para o cuidado na $\mathrm{ABS}$, ainda se encontram entraves para a realização da visita domiciliar. Nesta pesquisa, foi identificada como principal dificuldade para os enfermeiros a sobrecarga de tarefas na UBS, as quais consomem grande parte do tempo e, consequentemente, interferem na realização da visita domiciliar.

A visita domiciliar é um espaço que se a gente não brigar por ele, não acontece. Porque nós, enfermeiros, temos uma carga extremamente grande de atividade; se deixar, você só fica dentro da unidade. (E7)

As exigências de produtividade e o cadastramento ultrapassado, subestimando o número de famílias da área, colaboram para a sobrecarga de trabalho ${ }^{15}$. Devido ao número elevado de tarefas e o tempo limitado, muitos enfermeiros optam por realizar outras atividades, em detrimento das visitas domiciliares. Isso aponta para a necessidade de reflexão sobre que práticas devem ser priorizadas no conjunto de atividades.

É importante salientar que em diversas entrevistas, os enfermeiros relataram suas práticas profissionais cotidianas sem, contudo, denominá-las como cuidado. No entanto, identificou-se o ato de cuidar nas falas que abordavam relações pessoais de diálogo e escuta, valorização, humanização e respeito entre enfermeiros e usuários. Esta questão também reflete a necessidade de debater sobre como o enfermeiro desenvolve o seu cuidado na $A B S$ e acrescentar à discussão o papel do enfermeiro na ABS.

Embora o enfermeiro desenvolva diversas atividades voltadas para o cuidado na visita domiciliar, percebeu-se que ele tem realizado, em maior número, as atividades assistenciais, ou seja, as relacionadas à dimensão técnica. Assim, ele tem deixado de utilizar o espaço da visita domiciliar para fortalecer o vínculo com as famílias e promover a educação e saúde. No entanto, neste espaço, o enfermeiro visa atender às diferentes necessidades de saúde, preocupando-se desde a infraestrutura domiciliar até as relações familiares $^{16}$, e por isso incluindo as mais diversas abordagens e não somente as assistenciais.

\section{Atividades educativas}

As atividades educativas também foram identificadas como uma importante prática de cuidado dos enfermeiros da atenção básica, que se relacionam, principalmente, às orientações por eles prestadas aos diferentes atores sociais. Perpassam a promoção da saúde, a prevenção e o controle de agravos, o autocuidado, e ainda, as orientações técnicas sobre a realização de procedimentos. São realizadas por meio de consultas de enfermagem individuais ou coletivas, palestras e grupos de saúde, como constatado nos relatos a seguir:

[...] nossa prática, aqui, consiste na educação de nossos clientes, que nós atendemos; a questão das vacinas e da importância do cumprimento dos prazos, a gente agenda conforme as vacinas. (E16).

O grupão ocorre lá em cima, a gente vai fazer a palestra, explica para eles, vê o nível que cada usuário tem, vai depender muito do nível. O poder aquisitivo ébaixo, aquela coisa toda. Você renova sempre as palestras com eles, as palestras de como estão indo, se eles estão tomando o remédio direito, nas horas certas. (E11).

Os relatos apresentados parecem criar contradições referentes à prática da educação em saúde voltada para o cuidado. Se, por um lado, demonstram a possibilidade de um contato maior, mais interativo e zeloso com o usuário, por outro, revelam as dificuldades dos enfermeiros em concretizar tal educação em saúde, de forma que seja emancipadora, que haja a troca de saberes e, consequentemente, se aproxime do cuidado de enfermagem.

Existem formas de conhecer que incluem os conhecimentos populares e leigos que estariam do lado invisível da linha que separa a distinção científica entre verdadeiro e falso. As experiências tornadas invisíveis são desperdiçadas e desconsideradas como experiências legítimas de construção de saberes. Nesse sentido, reconhece-se a pluralidade de conhecimentos heterogêneos, que vão além do científico, e as interações sustentáveis e dinâmicas entre eles ${ }^{17}$. Para que ocorra interação entre esses saberes é preciso que ambas as partes, profissionais de saúde e usuários, estejam comprometidas em escutar e expressar suas opiniões.

Percebeu-se ainda, no contexto analisado das entrevistas, que, dentro dessas atividades educativas, há ênfase na dimensão técnica do cuidado, pois, mesmo ao se promover um diálogo/escuta, há um direcionamento preponderante aos aspectos biológicos, por vezes de maneira verticalizada.

[...] por mais que a gente fale do cuidado dos pés, eles insistem em usar sapato apertado, menor do que o número do pé, causando calcificação, causando ferida. (E23)

[...] a gente entrava com os cuidados do bebê, aí, eu orientava a mãe a dar o banho, tinha a fono que falava, a psicóloga. (E25)

Eu vejo o cuidado na minha prática, porque eu faço o curativo na cicatriz umbilical do bebê que chega na unidade e faço o cuidado no paciente, aplicando vacinas e medicamentos. É o cuidado na prática, e não só na teoria. (E26).

Identificou-se nos relatos que a dimensão técnica do cuidado perpassa, além das atividades educativas, pelas mais diversas atividades do enfermeiro na ABS, tais como aplicação de vacinas, curativo, aferição de sinais vitais, entre outros. Essas expressões indicam que os enfermeiros entendem o cuidado como ação ao executar um procedimento, indo ao encontro direto do indivíduo a ser cuidado. Mostram, ainda, a possibilidade do início de uma relação terapêutica 
entre o profissional de saúde e o indivíduo, pois, ao identificar o cuidado como um procedimento técnico, o enfermeiro amplia a possibilidade de interação e comunicação com o indivíduo que procura os serviços de saúde.

O avanço tecnológico tem contribuído para um cuidado centrado em procedimentos técnicos, ainda influenciados pelo modelo hegemônico tradicional, com práticas assistenciais mecanicistas e fragmentadas, que priorizam ações curativas. Porém, é preciso ir além do cuidado curativo, pois em um momento de cuidar não se pode esquecer a singularidade, a integralidade e o contexto de vida do indivíduo ${ }^{8}$. $\bigcirc$ cuidado é "sempre uma interação entre duas pessoas" ${ }^{18: 652}$ e, por isso, não se pode separar o procedimento técnico da relação interpessoal, uma vez que a tecnologia é controlada por pessoas.

Essas inferências levam à reflexão sobre a necessidade de instigar os profissionais enfermeiros a compreenderem o desenvolvimento do cuidado em suas práticas, seus significados e delimitações. Visto que cuidado, ainda que, por muito tempo desconsiderado como um saber, e percebido apenas como um fazer, parece estar hoje inserido nas práticas dos enfermeiros, mesmo quando não reconhecido. Inferiu-se, portanto, que o cuidado permeia e ao mesmo tempo é perpassado pelas diferentes práticas desenvolvidas, ainda que de modo indireto.

\section{Conclusão}

Ao pesquisar as práticas de cuidado desenvolvidas pelos enfermeiros na atenção básica do município do Rio de Janeiro, percebeu-se a sua diversidade, tais como visita domiciliar, consulta de enfermagem, atividades educativas, assistenciais e administrativas e acolhimento. No entanto, as que se destacaram foram as consultas de enfermagem, a visita domiciliar e as atividades educativas.

De modo geral, as práticas de cuidado dos enfermeiros na ABS estão voltadas para a dimensão técnica e para os aspectos biológicos, sendo que o cuidado expressivo/sensível apresenta-se pouco fortalecido, o que pode comprometer a atenção integral à saúde do usuário.

Foi possível identificar, por meio dos resultados obtidos, a complexidade da prática do enfermeiro, do cuidado que permeia essa prática e do fazer como ação diária. Todavia, percebe-se certa dificuldade, por parte desses profissionais, em definir claramente a presença do cuidado de enfermagem nas práticas que realizam no âmbito da ABS, o que demonstra a necessidade de debater esse tema e o papel do enfermeiro.

Aponta-se, como limitação da pesquisa, a restrição do campo de estudo em somente quatro CMS e duas UBSF da cidade do Rio de Janeiro, a qual, apesar de sua relevância, não possibilita generalizar os resultados para outros municípios, por constituir uma amostra específica.

Esta pesquisa indica a importância de que sejam ampliados os estudos sobre práticas de cuidado de enfermeiros voltadas para a atenção básica, identificando tanto o caráter objetivo quanto subjetivo dessas práticas. E, ainda, aprofundar a compreensão do papel desses profissionais nas práticas de cuidado e os sentidos que eles atribuem a tais práticas, e com isso, corroborar a relevância do cuidado de enfermagem na área da atenção básica.

\section{REFERÊNCIAS}

1.Polaro SHI, Gonçalves LHT, Alvarez AM. Construindo o fazer gerontológico pelas enfermeiras das Unidades de Estratégia Saúde da Família. Rev esc enferm USP. 2013; 47: 160-7.

2.Ferreira VA, Acioli S. O cuidado na prática do enfermeiro no campo da atenção primária em saúde: Produção científica. Rev enferm UERJ. 2009; 17: 506-9.

3.Bourdieu P. Esboço de uma teoria da prática. In: Ortiz R, organizador. Pierre Bourdieu: sociologia. São Paulo: Ática; 1994. p. 46-81.

4.Acioli S, Luz MT. Sentidos e valores de práticas populares voltadas para a saúde, a doença e o cuidado. Rev enferm UERJ. 2003; 11: 153-8.

5.Collière MF. Promover a vida. Lisboa (Pt): Lidel; 1999. 6. Waldow VR. Uma experiência vivida por uma cuidadora, como paciente, utilizando a narrativa literária. Texto contexto - enferm. 2011; 20: 825-33.

7.Fontes CAS, Alvim NAT. Cuidado humano de enfermagem: a cliente com câncer sustentado na prática dialógica da enfermeira. Rev enferm UERJ. 2008; 16: 193-9.

8.Serrano MTP, Costa ASMC, Costa NMVN. Cuidar em enfermagem: como desenvolver a(s) competência(s). Referência. 2011; 3(3): 15-23.

9.Waldow VR, Borges RF. Cuidar e humanizar: relações e significados. Acta Paul Enferm. 2011; 24: 414-8.

10.Kebian LVA, Acioli S. As diferentes práticas de cuidado na história da enfermagem em saúde pública brasileira. Rev enferm UFPE on line [site de Internet]. 2010 [citado em 6 ago 2014]. 4: 1124-30. Disponível em: http://www.revista.ufpe.br/revistaenfermagem/index. php/revista/article/download/1065/1652.

11.Bardin L. Análise de conteúdo. $6^{\mathrm{a}}$ ed. Lisboa $(\mathrm{Pt})$ : Edições 70; 2011.

12.Guerra CD, Dios MAA, Moral RR, García CJ, Torres LAP, Borrego MAR. Valoración de la satisfacción de usuarios de consulta de enfermería en centros de salud a partir de indicadores de calidad técnicos y de comunicación. Enferm glob. 2013; 12: 162-76.

13.Bernardes AG, Pelliccioli EC, Marques CF. Vínculo e práticas de cuidado: correlações entre políticas de saúde e formas de subjetivação. Ciênc saúde coletiva. 2013; 18: 2339-46.

14.Kawata LS, Mishima SM, Chirelli MQ, Pereira MJB, Matumoto S, Fortuna CM. Os desempenhos da enfer- 
meira na saúde da família: construindo competência para o cuidado. Texto contexto - enferm. 2013; 22: 961-70. 15.Trad LAB, Rocha AARM. Condições e processo de trabalho no cotidiano do Programa Saúde da Família: coerência com princípios da humanização em saúde. Ciênc saúde coletiva. 2011; 16: 1969-80.

16.Marin MJS, Gomes R, Siqueira Junior AC, Nunes CRR, Cardoso CP, Otani MP, et al. O sentido da visita domiciliária realizada por estudantes de medicina e enfermagem: um estudo qualitativo com usuários de unidades de saúde da família. Ciênc saúde coletiva. 2011; 16: 4357-65.

17. Santos BS, Meneses MP, organizadores. Epistemologias do Sul. São Paulo: Cortez; 2010.

18.Anéas TV, Ayres JRCM. Significados e sentidos das práticas de saúde: a ontologia fundamental e a reconstrução do cuidado em saúde. Interface - Comunic, Saude, Educ. 2011; 15: 651-62. 